\title{
La relación del balance sagital y la enfermedad del segmento adyacente
}

\author{
The relationship of sagittal balance and adjacent segment disease \\ Javier Andrés Martínez Silva, ${ }^{\star}$ Carlos Josué Victoria Buitimea, ${ }^{\ddagger}$ \\ Ricardo Luis Monge Romero,§ Gerardo Luis Pérez Palomares* \\ *Médico residente de tercer año de Ortopedia y Traumatología; $¥$ Médico adscrito Cirugía de Columna Vertebral; $\$$ Médico residente \\ de cuarto año de Ortopedia y Traumatología. Hospital General del Estado de Sonora.
}

\begin{abstract}
Resumen
La enfermedad del segmento adyacente se refiere al fenómeno de hipermovilidad inmediatamente después a un segmento rígido, el cual condiciona a radiculopatía o mielopatía. Los factores que propician la aparición de este fenómeno son múltiples, los más estudiados son la pérdida de movilidad segmentaria, alteración de la alineación sagital y cirugía multinivel. Existe evidencia que posterior a un procedimiento cervical es más importante la alineación segmental y no la global, lo cual afecta el resultado. En este caso se analiza la realización de una fusión anterior y su afección en la dinámica nativa por el cambio del balance sagital de $68 \mathrm{~mm}$, que sobrecarga la columna anterior y segmentos adyacentes, lo que remarca la correlación en la literatura de una evolución tórpida posterior a los $40 \mathrm{~mm}$ del eje sagital vertical. Dentro de la literatura hay pocos estudios que correlacionen la enfermedad del segmento adyacente y el balance sagital, en los cuales la correlación se establece de manera radiológica y no clínicamente, por lo cual se decide publicar este caso y agregar evidencia a esta correlación dando importancia a la planeación prequirúrgica para mantener y/o corregir el balance sagital cervical al realizar cirugía descompresiva y fusión para mielopatía cervical.
\end{abstract}

Palabras clave: Columna cervical, balance postural, calidad de vida, artrosis de columna, eje sagital cervical.

\begin{abstract}
Adjacent segment disease refers to the phenomenon of hypermobility of a level that comes immediately after a rigid segment that conditions a radiculopathy or myelopathy. Factors that predispose to this phenomenon are multiple, the more studied of which are loss of segmental mobility, sagittal balance alteration and multilevel surgery. There is evidence that after a cervical procedure, segmental and not global balance of the spine affect the outcome. In this clinical case study, we analyze the outcome after an ACDF and the impact on native dynamic properties after a change in SVA to $68 \mathrm{~mm}$ predisposing to an overload of the anterior column and adjacent segments that correlates with the actual literature of a poor outcome with an SVA over $40 \mathrm{~mm}$. There is a lack of high quality literature that correlates adjacent segmental disease and its correlation with sagittal balance that is only based on radiological findings and not clinical, for that reason we decided to publish this case to add to the growing evidence of this problem and the importance of good surgical planning taking account the maintenance and/or correction of cervical sagittal balance in decompressive and fusion surgery for cervical myelopathy.
\end{abstract}

Keywords: Cervical spine, postural balance, quality of life, osteoarthritis spine, cervical sagittal axis.

\section{Introducción}

Las funciones de la columna vertebral son: a) morfología (fenotipo cervical), b) protección (medular y de las raíces), c) movilidad, d) distribución de carga. Cuando cualquiera de estas funciones se ven comprometidas, nos encontramos ante un problema que a corto o largo plazo puede ocasionar manifestaciones clínicas, el canal cervical estrecho (CCE) presenta tres o cuatro funciones alteradas, lo se traduce en manifestaciones clínicas que repercuten en la calidad de vida. ${ }^{1-8}$

\section{Correspondencia:}

Javier Andrés Martínez Silva

E-mail: dr.javiermartinezsilva@gmail.com

Recibido: 14-05-2021. Aceptado: 23-05-2021.

Citar como: Martínez SJA, Victoria BCJ, Monge RRL, Pérez PGL. La relación del balance sagital y la enfermedad del segmento adyacente. Orthotips. 2021; 17 (3): 174-178. https://dx.doi.org/10.35366/100629 
El CCE puede presentarse en tres formas: radiculopatía espondilótica, mielopatía espondilótica o mielorradiculopatía espondilótica; es una patología frecuente en la práctica diaria que necesita eliminación de los sitios compresivos (estáticos y dinámicos), y estabilización del segmento, esto se puede realizar por: a) abordaje anterior (1. Discectomía y fusión cervical con caja o injerto óseo, 2. Corporectomía y colocación de jaula intersomática o injerto y placa, 3. Discectomía y colocación de prótesis de disco); b) abordaje posterior (1. Laminectomía e instrumentación posterior, 2. Laminoplastia); c) abordaje combinado anterior y posterior. Sin embargo, con cualquier tipo de procedimiento ya descrito la incidencia de enfermedad del segmento adyacente (ESA) se sigue presentando.

La enfermedad del segmento adyacente (ESA) se ha reportado a lo largo de la literatura universal, oscilando entre $0.6-9.6 \%$ en los casos donde se realiza artroplastia discal cervical (PCM, Prestige LP), y 1.4-14.6\% con discectomía y fusión cervical anterior. La presentación de esta complicación ha sido estudiada y los factores que la propician son variados, entre los más estudiados están: 1. Pérdida de la movilidad segmentaria (que aumenta las solicitaciones en el segmento suprayacente), 2. Alteración entre la alineación sagital y la cirugía multinivel (que modifica la distribución de cargas por la transición de movilidad de un segmento rígido a uno móvil). 9-13

Debido a la frecuencia de esta patología se presenta el caso y se describe la importancia e implicación del balance sagital (BS) en la historia natural de la enfermedad de la columna cervical.

\section{Presentación del caso}

Paciente femenino de 70 años de edad, con antecedente de hipertensión arterial en control,

Tabla 1: Sistema de clasificación de Nurick para la mielopatía cervical degenerativa.

Grado

0 No hay síntomas de la raíz o de la médula

I Signos o síntomas de las raíces. No hay evidencia de afectación de la médula

II Signos de afectación de la médula. Marcha normal

III Anomalía de la marcha. Capacidad para trabajar

IV La anomalía de la marcha impide el empleo

$\checkmark$ Puede deambular sólo con ayuda

VI En silla de ruedas o postrado en cama
Tabla 2: La escala modificada de la Asociación de Ortopedia Japonesa.

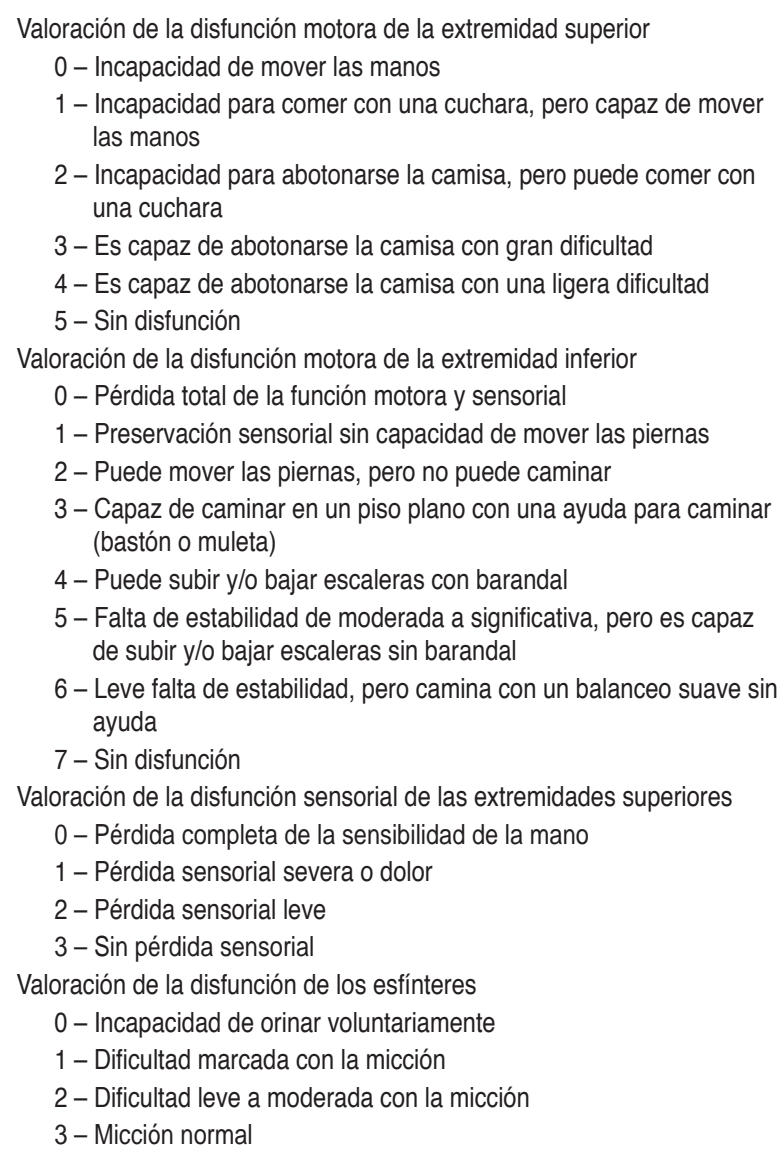

postoperada de discectomía cervical anterior más fusión intersomática con injerto autólogo con técnica de Cloward (1991).

Durante 20 años cursó de manera asintomática, en 2011 inicia con cervicalgia sin irradiación y tres meses después presenta irradiación a extremidad superior izquierda e interescapular con intensidad de siete puntos en escala visual análoga, mejoraba con reposo y se exacerbaba con los arcos de movilidad cervical, fue manejada en otra institución como pinzamiento subacromial, donde se realizó infiltración con corticosteroides y tuvo mejoría parcial; sin embargo, acude a institución hospitalaria para revaloración donde se valora paciente de la octava década de la vida con marcha atáxica, arcos de movilidad cervical limitados (extensión $30^{\circ}$, flexión $25^{\circ}$, inclinación $30^{\circ}$, rotación $45^{\circ}$ ), hiperestesia de C5-T1 izquierda, Spurling + izquierdo, Valsalva +, se sospecha de mielopatía espondilótica cervical severa y 

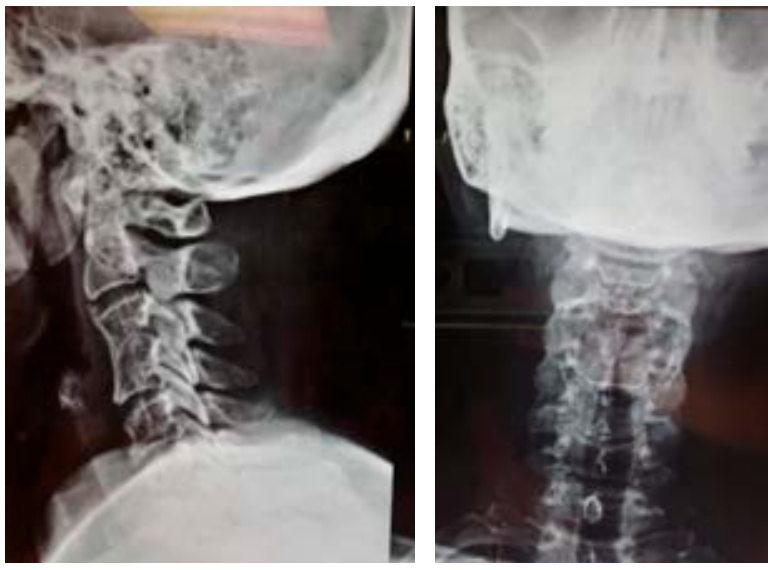

Figura 1: Radiografía anteroposterior y lateral de columna cervical.
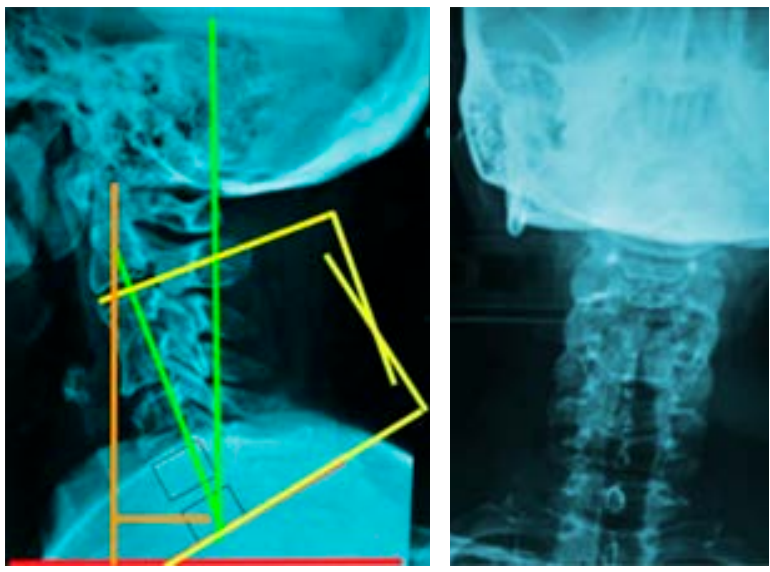

Figura 2: Radiografía anteroposterior y lateral de columna cervical con goniometría cervical. se aplica la escala de Nurick (IN) (Tabla 1) y la escala modificada de la Asociación de Ortopedia Japonesa (mJOA) (Tabla 2), donde se obtiene grado 3 de Nurick y 10 puntos de MJOA.

Se solicitan radiografías y resonancia magnética simple de columna cervical (Figuras 1 a 3 ): AP y lateral de columna cervical: rectificación de lordosis, osteofitos anteriores multisegmentarios, fusión del segmento C3C4, índice de Torg Pavlov 0.7, pendiente C7 30 , lordosis $\mathrm{C} 2-\mathrm{C} 79^{\circ}$, inclinación de $\mathrm{C} 22^{\circ}$, balance sagital cervical $6.8 \mathrm{~cm}$ RMN: rectificación de lordosis, fusión del segmento C3-C4, protrusión discal C2, C4, C5, C6, estenosis absoluta central $8 \mathrm{~mm}$, con sitio de mayor compresión C5-C6 además de compresión foraminal.

Por lo anterior, se decide realizar abordaje cervical anterior izquierdo tipo Smith-Robinson, discectomía C4, C5, C6, más colocación de caja PEEK y placa de tres segmentos con el objetivo de restaurar los ángulos variables del BS, estabilizar los segmentos involucrados y favorecer una fusión adecuada, en el postoperatorio inmediato se pudieron obtener los siguientes resultados (Figura 4).

- Pendiente C7 $23^{\circ}$.

- Lordosis C2-C7 $17^{\circ}$.

- Inclinación de C2 10.

- Balance sagital cervical $1.7 \mathrm{~cm}$.

- IN grado 0.

- mJOA 16 puntos.

\section{Discusión}

EI BS de la columna se ha relacionado con los síntomas, calidad de vida y dependencia de los
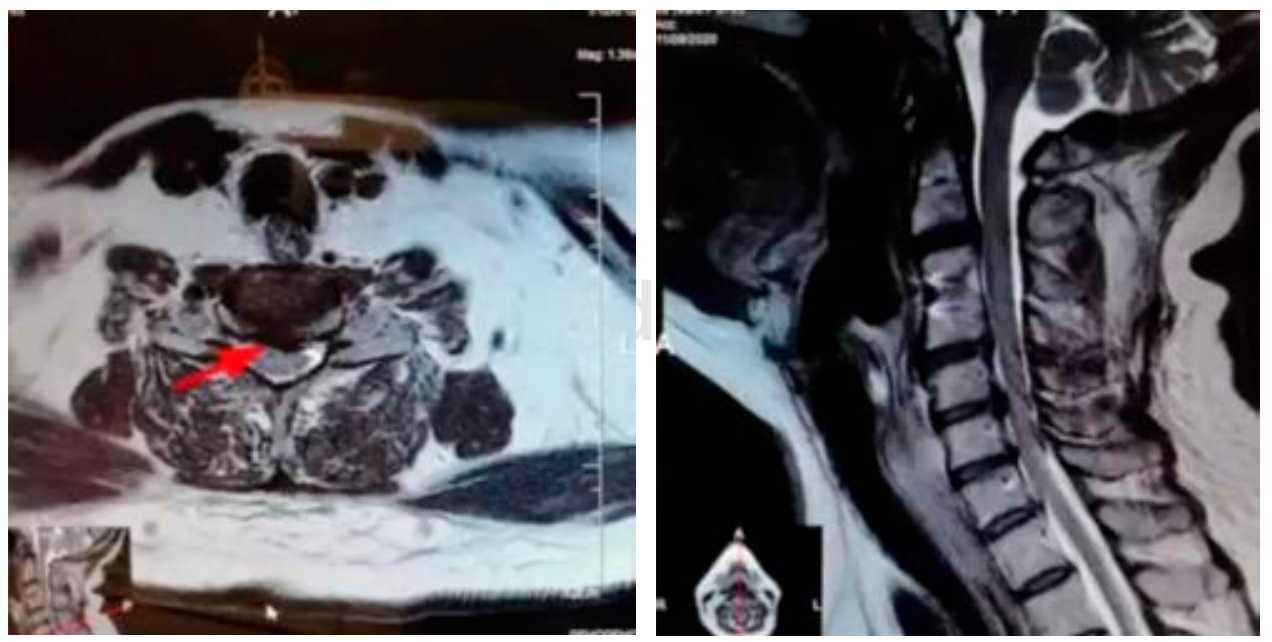

Figura 3:

Resonancia magnética nuclear simple (corte axial en segmento C6-C7 y corte sagital secuencia T2). 
pacientes, esto ha tomado importancia en el planeamiento quirúrgico para obtener mejores resultados funcionales a corto y largo plazo; sin embargo, no existe un consenso en los parámetros aceptados para cada medición en columna cervical, por lo cual sigue siendo un área de investigación futura.

Las mediciones que más han mostrado algún tipo de relevancia clínica son:

- Pendiente de C7/T1.

- Inclinación de C2.

- Lordosis cervical.

- Balance sagital.

La pendiente de C7 oT1 (valor normal $19.64 \pm 8.76^{\circ}$ ) es la base de la columna cervical y por ello se relaciona de manera directa proporcional con la lordosis. La inclinación de C2 (valor normal $12.84 \pm 5.6^{\circ}$ ) se relaciona de manera indirecta con la lordosis y directamente con el balance sagital. Con respecto a la lordosis cervical C2-C7 (valor normal $12.3 \pm 4.8^{\circ}$ ), se ha reportado que hasta $30 \%$ de la población no presenta lordosis cervical y no tiene sintomatología. Un aumento del balance sagital cervical $(4.5 \pm 2.6 \mathrm{~mm})$ se ha relacionado con el deterioro de la calidad de vida. ${ }^{11}$

Al tener conocimiento sobre la relación del BSC y los resultados funcionales en esta época de la cirugía moderna, es necesario establecer objetivos cuantitativos para mejorar la calidad de vida de los pacientes y disminuir la incidencia de problemas biomecánicos en segmentos adyacentes. ${ }^{14}$

Dentro de las múltiples causas de la enfermedad del segmento adyacente se ha relacionado la pérdida del BS y la progresión degenerativa de los mismos. ${ }^{15-19}$

El fundamento anatomopatológico es: la columna anterior se somete más a compresión y el complejo osteoligamentario posterior a tensión, lo que propicia un pinzamiento discal anterior y con ello la progresión artrósica del complejo triarticular de Kirkaldy-Willis. ${ }^{19}$ En el caso que se presenta podemos analizar cómo la fusión del segmento C3-C4 afectó la dinámica cervical segmentaría y global además de una pérdida del BS, lo que fomentó la degeneración de los segmentos infrayacentes por la zona de transición C3-C4 (estática) C4-C6 (dinámica) C7-T1 (estática). ${ }^{20}$

\section{Conclusiones}

La presentación de este caso busca ejemplificar la importancia de planificar y realizar cirugía con
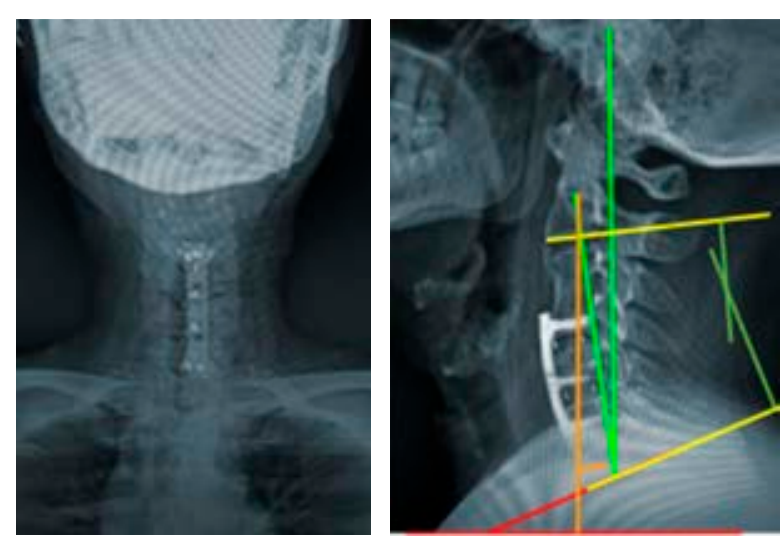

Figura 4: Radiografía anteroposterior y lateral de columna cervical con goniometría cervical control postquirúrgico inmediato.

objetivos cuantitativos para mejorar los resultados funcionales.

Es por eso que se recomienda respetar el BS para retrasar la historia natural de la enfermedad vertebral y con ello limitar la sintomatología y la tasa de cirugía de revisión.

\section{Referencias}

1. Lee SH, Son ES, Seo EM, Suk KS, Kim KT. Factors determining cervical spine sagittal balance in asymptomatic adults: correlation with spinopelvic balance and thoracic inlet alignment. Spine J. Spine J. 2015; 15 (4): 705-712.

2. Scheer JK, Tang JA, Smith JS, et al. Cervical spine alignment, sagittal deformity, and clinical implications: a review. J Neurosurg Spine. 2013; 19: 141-159.

3. Miyazaki M, Hymanson HJ, Morishita $\mathrm{Y}$, et al. Kinematic analysis of the relationship between sagittal alignment and disc degeneration in the cervical spine. Spine. 2008; 33: E870-E876.

4. Smith JS, Lafage V, Ryan DJ, et al. Association of myelopathy scores with cervical sagittal balance and normalized spinal cord volume: analysis of 56 preoperative cases from the AOSpine North America Myelopathy study. Spine. 2013; 38 (22, Suppl 1): S161-S170.

5. Guérin P, Obeid I, Gille O, et al. Sagittal alignment after single cervical disc arthroplasty. J Spinal Disord Tech. 2012; 25: 10-16.

6. Glassman SD, Bridwell K, Dimar JR, Horton W, Berven S, Schwab $F$. The impact of positive sagittal balance in adult spinal deformity. Spine. 2005; 30: 2024-2029.

7. Kawakami M, Tamaki T, Yoshida M, Hayashi N, Ando M, Yamada $\mathrm{H}$. Axial symptoms and cervical alignments after cervical anterior spinal fusion for patients with cervical myelopathy. J Spinal Disord. 1999; 12: 50-56.

8. Nurick $S$. The natural history and the results of surgical treatment of the spinal cord disorder associated with cervical spondylosis. Brain. 1972; 95 (1): 101-108.

9. Tracy JA, Bartleson JD. Cervical spondylotic myelopathy. Neurologist. 2010; 16 (3): 176-187.

10. Rhee JM, Heflin JA, Hamasaki T, Freedman B. Prevalence of physical signs in cervical myelopathy: a prospective, controlled study. Spine (Phila Pa 1976). 2009; 34 (9): 890-895. 
11. Harrop JS, Naroji S, Maltenfort M, et al. Cervical myelopathy: A clinical and radiographic evaluation and correlation to cervical spondylotic myelopathy. Spine (Phila Pa 1976). 2010; 35 (6): 620-624.

12. You J, Tang X, Gao W, Shen Y, Ding W-Y, Ren B. Factors predicting adjacent segment disease after anterior cervical discectomy and fusion treating cervical spondylotic myelopathy: a retrospective study with 5-year follow-up. Medicine (Baltimore). 2018; 97 (43): e12893.

13. Weinberg DS, Rhee JM. Cervical laminoplasty: indication, technique, complications. J Spine Surg. 2020; 6 (1): 290-301.

14. Kirkaldy-Willis WH, Farfan HF. Instability of the lumbar spine. Clin Orthop Relat Res. 1982; (165): 110-123.

15. De Haro Estrada JA. Balance sagital cervical [Internet]. Medigraphic.com. [cited 2021 Apr 19]. Available from: https:// www.medigraphic.com/pdfs/orthotips/ot-2015/ot153c.pdf.

16. Lebl DR, Hughes A, Cammisa FP Jr, O'Leary PF. Cervical spondylotic myelopathy: pathophysiology, clinical presentation, and treatment. HSS J. 2011; 7 (2): 170-178.

17. Park JH, Cho CB, Song JH, Kim SW, Ha Y, Oh JK. T1 slope and cervical sagittal alignment on cervical CT radiographs of asymptomatic persons. J Korean Neurosurg Soc. 2013; 53 (6): 356-359.

18. Tan LA, Riew KD, Traynelis VC. Cervical spine deformity-part 1: Biomechanics, radiographic parameters, and classification. Neurosurgery. 2017; 81 (2): 197-203.

19. Patel PD, Arutyunyan G, Plusch K, Vaccaro A Jr, Vaccaro AR. A review of cervical spine alignment in the normal and degenerative spine. J Spine Surg. 2020; 6 (1): 106-123.

20. Tetreault L, Kopjar B, Nouri A, et al. Escala modificada de la Asociación Ortopédica Japonesa: establecimiento de criterios para deterioro leve, moderado y grave en pacientes con mielopatía cervical degenerativa. Eur Spine J. 2017; 26 (1): 78-84.

\section{Conflicto de intereses}

Ninguno de los participantes de este manuscrito tiene conflicto de intereses. 